\title{
Therapeutic efficacy of traditional Chinese medicine, Shen-Mai San, in cancer patients undergoing chemotherapy or radiotherapy: study protocol for a randomized, double-blind, placebo-controlled trial
}

\author{
Lun-Chien Lo ${ }^{1,2}$, Chia-Yun Chen ${ }^{1,2}$, Shou-Tung Chen ${ }^{3,4}$, Hung-Chang Chen ${ }^{5}$, Tsung-Chieh Lee \\ and Cheng-Shyong Chang ${ }^{6 *}$
}

\begin{abstract}
Background: Cancer is one of the major health issues worldwide. An increasing number of cancer patients are offered treatment with surgery, chemotherapy and radiotherapy. Traditional Chinese medicine (TCM) is one of the most common complementary therapies offered to cancer patients in Taiwan. We designed a randomized, double-blind, placebo-controlled clinical trial to evaluate the efficacy of TCM in patients with cancer.

Methods/design: In this study, inclusion criteria are postoperative patients with histologically confirmed cancer within 3 years who are undergoing chemotherapy or radiotherapy, more than 18 years old, have given signed informed consent, have the ability to read Chinese, and the ability for oral intake.

Exclusion criteria include being pregnant, breast feeding, having completed chemotherapy or radiotherapy, brain metastasis with Eastern Cooperative Oncology Group (ECOG) performance status of two to four, delusion or hallucinations, acute infection, and have received medications under other clinical trials.

The patients were separated into an intervention group (Shen-Mai-San, SMS) and a placebo group for four weeks using a randomized, double-blind procedure. The European Organization for Research and Treatment of Cancer (EORTC) Quality of Life questionnaire (QOL-C30) was used to evaluate the quality of life. General data, hemoglobin $(\mathrm{Hb})$, hematocrit (Hct), glutamic-oxalacetic transaminase (GOT), glutamic-pyruvic transaminase (GPT), blood urea nitrogen (BUN), creatinine, carcinoembryonic antigen (CEA), TCM diagnosis data and heart rate variability (HRV) were also recorded. These data were collected at baseline, two weeks and four weeks after receiving medication. The patients were prescribed granules which contained therapeutic medicines or placebo. Paired-T test was used for statistical analysis.
\end{abstract}

Discussion: Shen-Mai-San is composed of processed Ginseng radis, Liriope spicata, and Schizandrae fructus. It was found to be effective for treating cancer-related fatigue and had anti-fatigue activity. In TCM theory, SMS has a synergistic effect for qi and yin deficiency and has the ability to prevent fatigue. The symptoms of qi and yin deficiency are similar to chemotherapy- or radiotherapy-induced side effects. In order to evaluate the efficacy of SMS on cancer treatment, we designed a randomized, double-blind, placebo-controlled trial.

Trial registration: This study is registered to Clinical Trails.gov NCT01580358

Keywords: Traditional Chinese Medicine, Cancer, Chemotherapy, Radiotherapy

\footnotetext{
* Correspondence: 15120@cch.org.tw

${ }^{6}$ Department of Hematology and Oncology, Changhua Christian Hospital,

135 Nanxiao St, Changhua City, Changhua Country 500, Taiwan

Full list of author information is available at the end of the article
} 


\section{Background}

Cancer has been the most horrible disease worldwide. In Taiwan, cancer has been ranked as the leading cause of death since 1982 [1]. Liver cancer, lung cancer and colon cancer are the leading cancers and account for $50 \%$ of all cancers in Taiwan [1]. The treatment of cancer includes surgery, chemotherapy, radiotherapy, target therapy, and so on. The choice of management depends on cancer type and stage. Treatment generally has a beneficial effect on curbing the progression of the disease, but sometimes causes disturbing side effects, such as nausea, vomiting [2], mucositis [3], general weakness [4] and so on which may decrease a patient's quality of life [5]. The use of complementary and alternative medicine (CAM) has increased over the past years around the world [6]. Some studies indicate that the use of CAM is found more frequently in patients with cancer than in the general population and the use of CAM is common in these patients $[7,8]$. The use of CAM increased among these patients who had a lower quality of life [9]. Many studies indicate an improvement in the quality of life and general health in cancer patients adopting CAM treatment $[10,11]$. CAM treatment includes dietary supplements, prayers, traditional Chinese herbs and botanicals [12].

Traditional Chinese medicine (TCM) is one of the most common CAM used in Taiwan. Some cancer patients search for a second opinion or TCM instead of regular treatment. Many patients in Taiwan turn to TCM due to poor quality of life or general weakness after surgery, radiotherapy or chemotherapy. Some studies reported that TCM did demonstrate some beneficial effects on maintaining immune function and liver protection in patients who were simultaneously receiving chemotherapy $[13,14]$. The purpose of this study is to evaluate the efficacy of TCM in improving the quality of life of cancer patients undergoing chemotherapy or radiotherapy. Although many CAMs have demonstrated positive effects on lessening the side effects suffered after chemotherapy or radiotherapy, a rigid validation using a randomized controlled trial (RCT) remains the best way to examine the effect of TCM in these patients.

\section{Methods/Design Design}

The study is conducted as a randomized, double-blind, placebo controlled trial to examine the effects of TCM in patients with cancer who are undergoing chemotherapy or radiotherapy. All participants are randomly assigned to an intervention or placebo group. The study procedures and informed consent form have been approved by the Institutional Review Board of Changhua Christian Hospital in Taiwan. (IRB no.070301). Figure 1 illustrates the flow diagram of the trial for both the intervention and placebo groups.

\section{Medication}

According to the guidelines for clinical trials of new Chinese medicine announced by the Department of Health, Executive Yuan in Taiwan [15], only formulae recorded in ancient TCM books may be used for clinical trials in Taiwan. The formula employed in this trial, Shen-Mai-San (SMS) has a long history of application to disease treatment in China starting in the 12th century [16]. SMS is composed of three herb ingredients, namely Ginseng radis, Liriope spicata, and Schizandrae fructus. It was initially used for the treatment of a dual deficiency of qi and yin with symptoms of thirst and general weakness while suffering from heat stroke, fatigue, and so on [17]. According to TCM theory, SMS can reduce fatigue, thirst, general weakness, and so on. SMS is widely used now to treat patients with cardiac disease, fatigue and cancer $[18,19]$. Many patients have symptoms of general weakness, thirst, and fatigue after chemotherapy or radiotherapy. These symptoms are similar to the syndrome of qi and yin deficiency in TCM theory. In order to evaluate the efficacy of SMS in cancer patients after chemotherapy and radiotherapy, a randomized, double-blind, placebo-controlled trial will be performed.

SMS is manufactured as a concentrated herbal extract, packed $0.5 \mathrm{~g}$ per capsule, and labeled by Sun-Ten, a pharmaceutical company in Taiwan with good manufacturing practice (GMP). The placebo capsules are prepared with $0.5 \mathrm{~g}$ starch inside the same colored and sized capsules.

\section{Participants}

Patients will be recruited from the outpatients of the Oncology and Chinese Medicine Department in ChangHua Christian Hospital, a medical center in central Taiwan. Sixty patients will be recruited for the study and divided into an intervention group and a placebo group.

\section{Inclusion criteria}

In this study, inclusion criteria are as follows: (1) postoperative patients with histologically confirmed cancer within 3 years who are undergoing chemotherapy or radiotherapy; (2) more than 18 years old; (3) signed informed consent; (4) able to read Chinese; and (4) have the ability to take medicine by mouth.

\section{Exclusion criteria}

Exclusion criteria include: (1) being pregnant; (2) breast feeding; (3) had completed chemotherapy or radiotherapy; (4) brain metastasis with Eastern Cooperative Oncology Group (ECOG) performance status of two to four; (5) suffering from delusion or hallucination; (6) acute infection; and (7) had received medications for other clinical trials. 


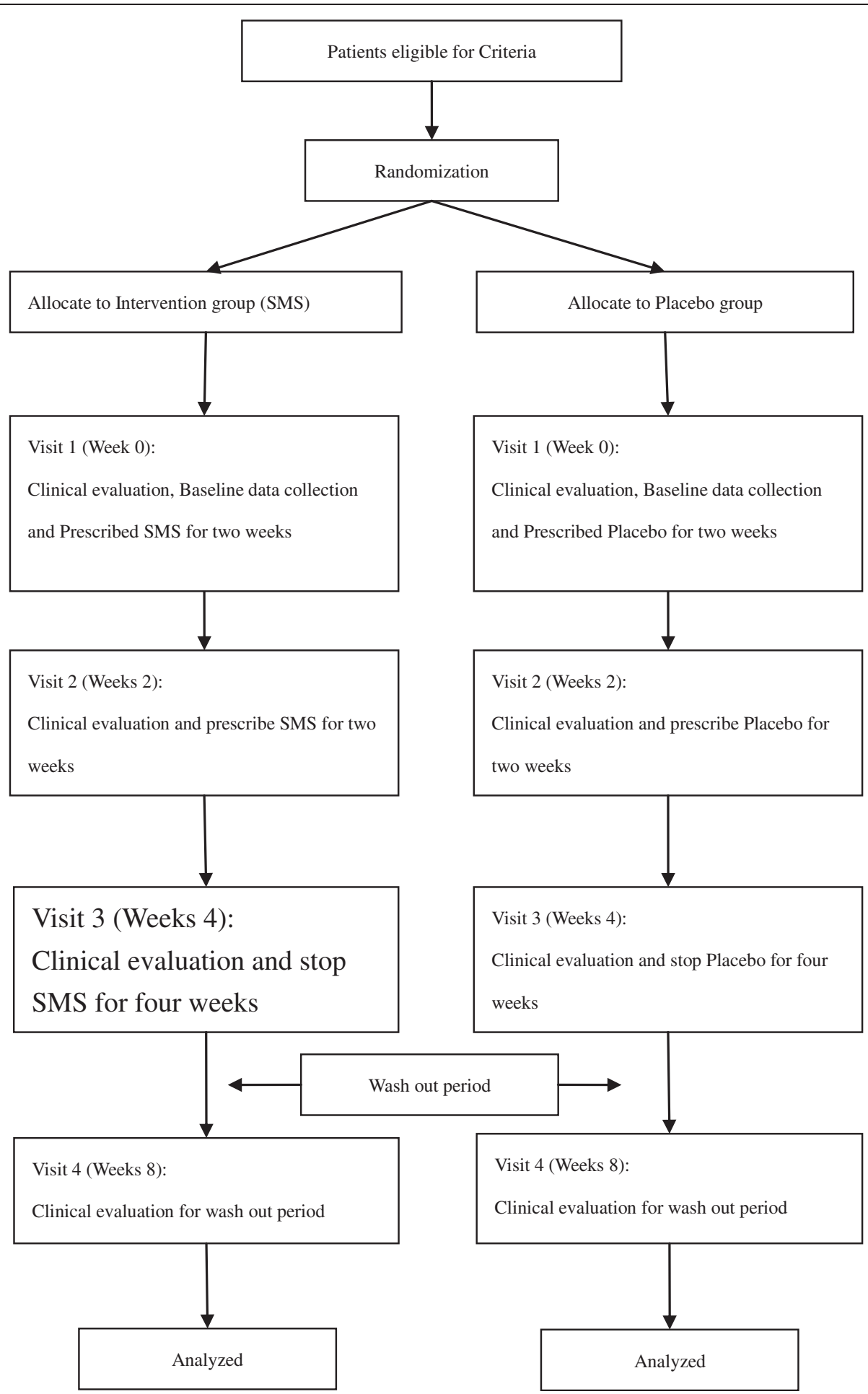

Figure 1 TCM therapeutic efficacy trial flow chart. 


\section{Randomization}

Patients who meet the inclusion criteria will be randomly assigned to an intervention group (SMS) or placebo group by the clinical research coordinator (CRC).

\section{Blinding}

The patients will receive capsules either with SMS or placebo. These capsules are the same in appearance, color and shape. The researchers and patients will not know which group the patients are allocated to from the appearance of the medication given.

\section{Intervention}

One experienced TCM physician will be assigned to record the general data, TCM diagnostic data and prescribed medication for two weeks at the first visit. The patients will take the prescription to the pharmacy and the CRC will give either SMS or placebo in a capsule containing either $0.5 \mathrm{mg}$ medication or starch. Patients will take eight capsules three times a day. The intervention period will be four weeks. Two weeks and four weeks after the first visit, patients will come back to out-patient-department (OPD) for clinical evaluation and medication.

After four weeks on the prescribed medication, the patients will stop taking SMS and placebo for another four weeks and then come back to OPD for clinical evaluation.

\section{Data collection}

We will collect basic data such as gender, age, cancer type, stage, and metastasis or no metastasis at base line. Other than the basic data, such as gender and age, laboratory data, for example, WBC, Hb, Hct, CEA, platelet and HRV will be collected. GOT, GPT, BUN, and creatinine will also be recorded to facilitate the evaluation of liver and renal functions.

\section{Statistical analysis}

Statistical analysis will be performed by the Department of Mathematics and the Institute of Statistics and Information Science, National Changhua University of Education in Taiwan. We will use the statistical software $\mathrm{R}$ to analyze the data. Paired-T test will be derived for statistically analysis.

\section{Primary outcome}

Quality of life is evaluated multi-dimensionally using the Taiwan Chinese Version of the EORTC Quality of Life Questionnaire C30 (QLQ-C30). The patients will complete the questionnaire at baseline (first visit), two weeks later (second visit), four weeks later (third visit) and eight weeks later (fourth visit). The score will be calculated and compared within group and between groups before and after medication.
The laboratory data collected will be also used to evaluate the general health status and monitor the liver and renal functions to assess the safety of SMS.

\section{Discussion}

There is an ever increasing number of patients suffering from cancer every year. Although various types of treatment are performed on a routine basis, surgery, chemotherapy and radiotherapy remain the major modes of cancer treatment. Through chemotherapy or radiotherapy, not only tumor cells, but also normal cells, are simultaneously killed. Many side effects, such as lower white blood cell count, general weakness and loss of appetite are often observed during and after the treatment. These side effects may lower the quality of life of these patients.

Many CAMs show significant improvements in chemotherapy- or radiotherapy-related side effects and TCM is the most common means of CAM in Taiwan. One study reported utilizing TCM to improve the quality of life in women undergoing chemotherapy for ovarian cancer and the result showed no significant difference in global health status but there was less neutropenia [13]. Another study used TCM for patients with colon or breast cancer requiring chemotherapy; there was no significant difference in hematologic toxicity but there was a significant impact on the control of nausea [20]. We think that we can select formulae according to the most common symptoms of the patients using TCM theory and lessen the side effects caused by chemotherapy in cancer treatment.

SMS is used for the treatment of myocardial infarction (MI) and has a protective effect for renal ischemic damage during MI $[19,21]$. Some studies also reported that SMS can improve sperm viability and sperm movement parameters in vitro [22]. According to ancient TCM theory, SMS could also prevent fatigue in the general population and especially when used in patients with qi and yin deficiency. It is composed of processed G. radis, L. spicata, and S. fructus. Ginseng was reported to have some activity and tolerable toxicity to cancer-related fatigue [23]. Ginseng polysaccharides also had anti-fatigue activity in a study in mice [24]. L. spicata was used for yin deficiency according to TCM theory and had hypoglycemic and hypolipidemic potential for type 2 diabetes[25]. S. fructus was used for the benefit of qi and promoted body fluid production according to TCM theory and was also reported to have effects in viral- and chemical-induced hepatitis [26].

All ingredients of SMS are considered to improve side-effects induced by chemotherapy or radiotherapy, as described above under TCM theory. In addition to the described effects of every ingredient of SMS, the formula also has a synergic effect not yet realized. Many 
studies have shown that TCM formulae exhibit more significant synergistic effects than all the ingredients combined $[27,28]$. Moreover, the safety of concentrated herbal extracts is also important in this study. Many studies have shown that herbs may cause liver or renal damage $[29,30]$. The concentrated herbal extracts used in this study are produced by a GMP pharmaceutical company in Taiwan. Due to the strict flow control during the manufacturing process, the concentrated herbal extracts are highly homogeneous with good quality control, which is very different from those obtained using traditional methods. Therefore, the concentrated herbal extracts are considered safer than the traditional herbs.

More efforts will be exerted to prove the efficacy of TCM in helping patients with cancer through ongoing studies. Future researchers will focus on finding effective TCM formulae to help patients ease the side effects of chemotherapy and radiotherapy.

\section{Trial status}

At the time of manuscript submission, we have recruited thirty-six patients and are still recruiting patients.

\begin{abstract}
Abbreviations
BUN: Blood urea nitrogen; CAM: Complementary and alternative medicine; CEA: Carcinoembryonic antigen; CRC: Clinical research coordinator; ECOG: Eastern Cooperative Oncology Group; EORTC: European Organization for Research and Treatment of Cancer; GMP: Good manufacturing practice; Hb: Hemoglobin; Hct: Hematocrit; HRV: Heart rate variability; GOT: Glutamic-oxalacetic transaminase; GPT: Glutamic-pyruvic transaminase; OPD: Out-patient-department; QLQ-C30: Quality of Life Questionnaire C30; SMS: Shen-Mai-San; TCM: Traditional Chinese medicine.
\end{abstract}

\section{Competing interests}

The authors declare that they have no competing interests.

\section{Authors' contributions}

CSC, LLC, CYC, STC, HCC and TCL all contributed to the development of the study protocol. LLC was the principal investigator and managed the protocol. CYC was involved in the initial draft of the manuscript and writing it. CSC and LLC were involved in reviewing the manuscript. All authors read and approved the final manuscript.

\section{Acknowledgements}

Funding has been received from the committee on Chinese Medicine and Pharmacy, Department of Health, Executive Yuan in Taiwan. The authors acknowledge doctors for referring participants to the project in Changhua Christian Hospital.

\footnotetext{
Author details

'Department of Chinese Medicine, Changhua Christian Hospital, 135 Nanxiao St, Changhua City, Changhua Country 500, Taiwan. ${ }^{2}$ Department of Mathematics and Institute of Statistics and Information Science, National Changhua University of Education, No.1, Jin-De Road, Changhua City, Changhua Country 500, Taiwan. ${ }^{3}$ Comprehensive Breast Cancer Center, Changhua Christian Hospital, 135 Nanxiao St, Changhua City, Changhua Country 500, Taiwan. ${ }^{4}$ Division of General Surgery, Department of Surgery, Changhua Christian Hospital, 135 Nanxiao St, Changhua City, Changhua Country 500, Taiwan. ${ }^{5}$ Division of Colorectal Surgery, Department of Surgery, Changhua Christian Hospital, 135 Nanxiao St, Changhua City, Changhua Country 500, Taiwan. ${ }^{6}$ Department of Hematology and Oncology, Changhua Christian Hospital, 135 Nanxiao St, Changhua City, Changhua Country 500, Taiwan.
}

Received: 15 June 2012 Accepted: 14 November 2012

Published: 3 December 2012

\section{References}

1. Chen CJ, You SL, Lin LH, Hsu WL, Yang YW: Cancer epidemiology and control in Taiwan: a brief review. Jpn J Clin Oncol 2002, 32(Suppl):S66-81.

2. Hesketh PJ: Chemotherapy-induced nausea and vomiting. N Engl J Med 2008, 358:2482-2494.

3. Valks R, Garcia-Diez A, Fernandez-Herrera J: Mucocutaneous reactions to chemotherapy. J Am Acad Dermatol 2000, 42:699.

4. Stone P, Richardson A, Ream E, Smith AG, Kerr DJ, Kearney N: Cancer-related fatigue: inevitable, unimportant and untreatable? Results of a multi-centre patient survey Cancer Fatigue Forum. Ann Oncol 2000, 11:971-975

5. Gupta D, Lis CG, Grutsch JF: The relationship between cancer-related fatigue and patient satisfaction with quality of life in cancer. $J$ Pain Symptom Manage 2007, 34:40-47.

6. Horneber M, Bueschel G, Dennert G, Less D, Ritter E, Zwahlen M: How many cancer patients use complementary and alternative medicine: a systematic review and metaanalysis. Integr Cancer Ther 2012, 11:187-203.

7. Mao JJ, Palmer CS, Healy KE, Desai K, Amsterdam J: Complementary and alternative medicine use among cancer survivors: a population-based study. J Cancer Surviv 2011, 5:8-17.

8. Lim CM, Ng A, Loh KS: Use of complementary and alternative medicine in head and neck cancer patients. J Laryngol Otol 2010, 124:529-532.

9. Wyatt G, Sikorskii A, Wills CE, Su H: Complementary and alternative medicine use, spending, and quality of life in early stage breast cancer. Nurs Res 2010, 59:58-66.

10. Sewitch MJ, Rajput Y: A literature review of complementary and alternative medicine use by colorectal cancer patients. Complement Ther Clin Pract 2010, 16:52-56.

11. Chen $Y Z$, Li ZD, Gao F, Zhang Y, Sun H, Li PP: Effects of combined Chinese drugs and chemotherapy in treating advanced non-small cell lung cancer. Chin J Integr Med 2009, 15:415-419.

12. Klafke N, Eliott JA, Wittert GA, Olver IN: Prevalence and predictors of complementary and alternative medicine (CAM) use by men in Australian cancer outpatient services. Ann Oncol 2012, 23:1571-1578.

13. Chan KK, Yao TJ, Jones B, Zhao JF, Ma FK, Leung CY, Lau SK, Yip MW, Ngan $H Y$ : The use of Chinese herbal medicine to improve quality of life in women undergoing chemotherapy for ovarian cancer: a double-blind placebo-controlled randomized trial with immunological monitoring. Ann Oncol 2011, 22:2241-2249.

14. Liu ML, Chien LY, Tai CJ, Lin KC: Effectiveness of traditional Chinese medicine for liver protection and chemotherapy completion among cancer patients. Evid Based Complement Alternat Med 2011, 2011:291843.

15. Guide Line of Clinical Trial of New Chinese Medicine. http://www.ccmp. gov.tw/public/public.asp?selno $=1254 \&$ relno $=1254 \&$ level $=C$.

16. Zhang $Y$, Zheng $H$, Zhang $Y$, Zhang $Y$, Zhang $Y$ : Zhang Yuansu yi xue quan shu. Beijing Shi: Zhongguo Zhong yi yao chu ban she; 2006.

17. World Health Organization. Regional Office for the Western Pacific Region: WHO international standard terminologies on traditional medicine in the Western Pacific Region. Manila, Philippines: World Health Organization, Western Pacific Region; 2007.

18. Liu P, Cao Y, Qiao X: Clinical study on shenmai injection in promoting postoperative recovery in patients of breast cancer. Zhongguo Zhong $X_{i}$ Yi Jie He Za Zhi 2000, 20:328-329.

19. Xu N, Qiu C, Wang W, Wang Y, Chai C, Yan Y, Zhu D: HPLC/MS/MS for quantification of two types of neurotransmitters in rat brain and application: myocardial ischemia and protection of Sheng-Mai-San. J Pharm Biomed Anal 2011, 55:101-108.

20. Mok TS, Yeo W, Johnson PJ, Hui P, Ho WM, Lam KC, Xu M, Chak K, Chan A, Wong $\mathrm{H}$, Mo F, Zee B: A double-blind placebo-controlled randomized study of Chinese herbal medicine as complementary therapy for reduction of chemotherapy-induced toxicity. Ann Oncol 2007, 18:768-774.

21. Lee IY, Lee CC, Chang CK, Chien CH, Lin MT: Sheng mai san, a Chinese herbal medicine, protects against renal ischaemic injury during heat stroke in the rat. Clin Exp Pharmacol Physiol 2005, 32:742-748.

22. Zhang F, Xu JX, Ma HG, Zhou LY, Cheng ZL: Sheng Mai Zhusheye improves the viability and movement parameters of human sperm in vitro. Zhonghua Nan Ke Xue 2009, 15:468-471. 
23. Barton DL, Soori GS, Bauer BA, Sloan JA, Johnson PA, Figueras C, Duane $S$, Mattar B, Liu H, Atherton PJ, Christensen B, Loprinzi CL: Pilot study of Panax quinquefolius (American ginseng) to improve cancer-related fatigue: a randomized, double-blind, dose-finding evaluation: NCCTG trial N03CA. Support Care Cancer 2010, 18:179-187.

24. Wang J, Li S, Fan Y, Chen Y, Liu D, Cheng H, Gao X, Zhou Y: Anti-fatigue activity of the water-soluble polysaccharides isolated from Panax ginseng C A. Meyer. J Ethnopharmacol 2010, 130:421-423.

25. Chen X, Bai X, Liu Y, Tian L, Zhou J, Zhou Q, Fang J, Chen J: Anti-diabetic effects of water extract and crude polysaccharides from tuberous root of Liriope spicata var. prolifera in mice. J Ethnopharmacol 2009, 122:205-209.

26. Liu GT: Pharmacological actions and clinical use of fructus schizandrae. Chin Med J (Engl) 1989, 102:740-749.

27. Lau KM, Lai KK, Liu CL, Tam JC, To MH, Kwok HF, Lau CP, Ko CH, Leung PC, Fung KP, Poon SK, Lau CB: Synergistic interaction between Astragali Radix and Rehmanniae Radix in a Chinese herbal formula to promote diabetic wound healing. J Ethnopharmacol 2012, 141:250-256.

28. Zhang A, Sun H, Yuan Y, Sun W, Jiao G, Wang X: An in vivo analysis of the therapeutic and synergistic properties of Chinese medicinal formula YinChen-Hao-Tang based on its active constituents. Fitoterapia 2011, 82:1160-1168.

29. Shaohua Z, Ananda S, Ruxia Y, Liang R, Xiaorui C, Liang L: Fatal renal failure due to the Chinese herb "GuanMu Tong" (Aristolochia manshuriensis): autopsy findings and review of literature. Forensic Sci Int 2010, 199:e5-7.

30. Takikawa H, Murata Y, Horiike N, Fukui H, Onji M: Drug-induced liver injury in Japan: an analysis of 1676 cases between 1997 and 2006. Hepatol Res 2009, 39:427-431.

doi:10.1186/1745-6215-13-232

Cite this article as: Lo et al:: Therapeutic efficacy of traditional Chinese medicine, Shen-Mai San, in cancer patients undergoing chemotherapy or radiotherapy: study protocol for a randomized, double-blind, placebo-controlled trial. Trials 2012 13:232.

\section{Submit your next manuscript to BioMed Central and take full advantage of:}

- Convenient online submission

- Thorough peer review

- No space constraints or color figure charges

- Immediate publication on acceptance

- Inclusion in PubMed, CAS, Scopus and Google Scholar

- Research which is freely available for redistribution 